\title{
Balance trabajo-familia: Cultura, nivel de conflicto y voluntad de permanencia en la empresa
}

\begin{abstract}
Elsa Del Castillo Mory
Doctora en Economía y Dirección de Empresas por la Universidad de Deusto, Bilbao/

San Sebastián, España. Decana de la Escuela de Postgrado de la Universidad

del Pacífico, Lima, Perú. Profesora principal e investigadora del Departamento

Académico de Administración de la Universidad del Pacífico, Lima, Perú.

Rosa María Fuchs Ángeles

International M.B.A. por el Instituto de Empresa Business School, Madrid-España.

Profesora e investigadora del Departamento Académico de Administración de la

Universidad del Pacífico, Lima, Perú.

Sandra Vera Linares

Administradora de Empresas por la Universidad del Pacífico, Lima-Perú. Asistente de

Decanato de la Escuela de Postgrado de la Universidad del Pacífico, Lima-Perú.

Amaia Arizkuren Eleta

Doctora en CC. EE. y Empresariales por la Universidad de Deusto, España. Profesora

Titular de la Facultad de CC. EE. y Empresariales y de la Escuela de Turismo de la Universidad de Deusto, San Sebastián, España.

Tanuja Agarwala

Doctora en Gestión y Postgrado en Psicología, Universidad de Delhi, India. Profesora asociada de la Facultad de Management Studies de la Universidad de Delhi, India.
\end{abstract}

\section{Resumen}

El balance trabajo-familia ha sido ampliamente estudiado por la literatura referida a la gestión humana. La búsqueda de dicho balance está asociada a prácticas de retención del talento. Diversos autores anotan que la existencia de políticas corporativas sobre este tema no resulta suficiente para reducir el conflicto que experimentan los trabajadores por las presiones laborales y familiares. Según afirman, se requerirá de una cultura que acepte y apoye el balance trabajofamilia. Esta investigación empírica sugiere que, en el contexto de las empresas peruanas del sector industrial, financiero y de telecomunicaciones en el que trabajan los 96 encuestados, una cultura organizacional que apoya el uso de estas prácticas reduce el conflicto que experimenta el trabajador y, más aún, ambas variables — cultura y conflicto- permiten estimar su voluntad de permanencia en la empresa. No obstante, los resultados obtenidos también sugieren que, si bien el conflicto familia-trabajo (FWC) guarda una relación negativa con la voluntad de 
permanencia en la empresa, el conflicto trabajo-familia (WFC) no muestra esta misma significancia cuando aparece una cultura que apoya este balance.

\section{Palabras clave:}

Balance trabajo-familia, cultura organizacional, conflicto trabajo-familia, permanencia en la empresa

\section{I) Introducción}

El presente trabajo de investigación ha centrado su atención en las tensiones que se presentan en el trabajador cuando desea o debe cumplir, de manera simultánea, responsabilidades que emanan de su rol laboral y de su rol como parte de una familia. Las presiones de la competencia y la exigencia por parte de los directivos para que los colaboradores dediquen cada vez más tiempo a su trabajo, invadiendo incluso su vida familiar, están propiciando que el trabajador experimente una sensación de conflicto entre sus objetivos laborales y sus objetivos personales. Por otro lado, en la sociedad moderna, las personas desean y deben asumir un rol más activo en el núcleo familiar, lo que también genera presión en el trabajador. Estas tensiones los llevan a enfrentarse con conflictos que una organización puede buscar reducir. La literatura da cuenta de la importancia del rol del directivo en la construcción de una cultura corporativa que favorezca la reducción de este tipo de conflictos. Por ello, de acuerdo con Lingard et al. (2007), la generación de iniciativas para conciliar la vida laboral y la vida familiar puede aliviar el nivel de conflicto que los trabajadores experimentan en su trabajo y en su vida personal (Warren y Johnson 1995; VanRijswick et al 2004 en Lingard et al. 2007) y, por otra parte, puede facilitar un mejor equilibrio y calidad de vida (Tausig y Fenwick 2001; Madsen 2003 en Lingard et al. 2007). Como consecuencia de ello, puede reducirse el riesgo de que estos profesionales tomen la decisión de desvincularse de la empresa para buscar soluciones en otro empleo.
Sandra Vera Linares - Amaia Arizkuren Eleta - Tanuja Agarwala

\section{II) Objetivos de investigación}

Esta investigación busca analizar la relación existente entre tres variables centrales en el tema del equilibrio trabajo-familia: la cultura organizacional que apoya las prácticas de balance; el conflicto que se produce entre las demandas y presiones de ambos mundos (expresado en el conflicto trabajo-familia [WFC] y el conflicto familia-trabajo [FWC]) y el deseo de permanencia del trabajador en su centro de trabajo. En ese sentido, se han establecido tres objetivos de investigación:

- Identificar el impacto que la cultura organizacional que apoya el equilibrio trabajo-familia tiene en el conflicto que experimenta el trabajador.

- Identificar el impacto que el conflicto trabajo-familia WFC y el conflicto familiatrabajo FWC tienen en la voluntad de permanencia en la empresa.

- Identificar el impacto que, tanto la cultura organizacional que apoya el equilibrio trabajo-familia, como los conflictos trabajo-familia y familia-trabajo tienen en la voluntad de permanencia del trabajador en la empresa.

\section{III) Marco Teórico}

\section{Cultura organizacional y conflicto en el balance trabajo-familia}

\section{El rol de la cultura organizacional}

De acuerdo con Thompson et al. (1999, en Mauno et al. 2005: 117-118), una cultura trabajo-familia se define como «los supuestos, las creencias y los valores compartidos con respecto a la medida en que la organización apoya y valora la integración de la vida laboral y familiar de los trabajadores».

Alles (2000) remarca la importancia de buscar como objetivo el equilibrio de los roles del trabajador, en contraposición a una mirada de enfrentamiento o competencia entre los mismos. Según afirma, esta preocupación de la empresa 
debiera traducirse no solo en un esfuerzo por promover un entorno cooperador, sino en trabajar con las actitudes de los miembros de la organización para que las políticas surtan efecto. En este sentido, los sistemas de valores de las organizaciones resultarán relevantes cuando las empresas busquen ser equitativas en la asignación de roles y en la exigencia laboral entre los miembros de su equipo humano (Kabanoff et al. 1995).

Más aún, la cultura organizacional puede influenciar la percepción que se tiene sobre el uso de prácticas de balance trabajo-familia (Lewis 2001 en O'Brien y Hayden 2008). Según Thompson et al. (1999, en Mauno et al. 2005), un primer componente de esta cultura trabajo-familia es el apoyo administrativo, concepto que se refiere al apoyo social y a la sensibilidad mostrada por los directivos frente a las responsabilidades familiares de los trabajadores. Este apoyo se ve reflejado en las actitudes y los valores de los jefes directos y de los gerentes de recursos humanos, así como en su comportamiento para alentar o desalentar la participación de los trabajadores en los programas de apoyo trabajo-familia. En general, los líderes, gerentes y jefes directos serán vistos como figuras clave para crear e influir en la cultura organizacional. Por otra parte, también podrán ser capaces de reducir el conflicto trabajo-familia que experimentan sus trabajadores (Mauno et al. 2005).

La segunda dimensión de la cultura, planteada por Thompson et al. (1999, en Mauno et al. 2005) se refiere al posible efecto en las oportunidades de desarrollo de carrera

como consecuencia de la utilización de beneficios trabajo-familia. En este sentido, se ha sugerido que la participación en los programas de trabajofamilia hace «menos visible» a un empleado en el trabajo, lo que puede afectar negativamente el desarrollo profesional y sus posibilidades de promoción. Por otra parte, los trabajadores que utilizan acuerdos de trabajo- familia pueden ser percibidos como menos comprometidos con su organización y el trabajo (Mauno et al. 2005).

Finalmente, una tercera dimensión de la cultura organizacional en apoyo a las prácticas de balance
Sandra Vera Linares - Amaia Arizkuren Eleta - Tanuja Agarwala

trabajo-familia, propuesta por Thompson et al. (1999, en Mauno et al. 2005), analiza las demandas de tiempo exigido al trabajador por parte de la empresa, expresado en las normas sobre el número de horas que cada trabajador debe dedicar al trabajo $\mathrm{o}$ a las actividades relacionadas con el trabajo. Si bien, por un lado, trabajar largas horas refleja participación, compromiso y productividad; por otro lado, este comportamiento puede ser un obstáculo para satisfacer las necesidades de la familia (Bailyn 1993, en Mauno et al. 2005). De acuerdo con Lewis (1999), la cultura de largas horas reduce la sensación de tener derecho a trabajar de forma compatible con la vida familiar. Como resultado, es probable que se origine el conflicto trabajofamilia. El conflicto trabajo-familia, a su vez, afecta al bienestar de un trabajador en diferentes ámbitos de la vida (Mauno et al. 2005), como se discutirá más adelante.

Burke (2010) realizó un estudio para determinar el nivel de beneficio obtenido por los hombres de negocios, cuando existen valores organizacionales que apoyan el balance trabajofamilia. En este estudio, analizó la relación existente entre las percepciones de hombres profesionales sobre los valores organizacionales que soportan un compromiso balanceado hacia su vida laboral y personal, y su efecto en el nivel de satisfacción personal y laboral, así como en su bienestar psicológico. El estudio arrojó que los hombres que encontraron mayor apoyo por parte de sus empresas, reportaron trabajar menos horas a la semana y disfrutar el trabajo. Asimismo, mostraron menos estrés laboral y mayor satisfacción con sus trabajos y sus carreras, menos deseos de renunciar, más satisfacción con sus amigos y con la comunidad y, finalmente, mayores niveles de bienestar emocional.

\section{El conflicto en el balance trabajo-familia}

El conflicto trabajo-familia se ha definido como "una forma de conflicto inter-roles en el que las presiones de los roles de los ámbitos del trabajo y la familia son incompatibles entre sí en algunos aspectos» (Greenhaus y Beutell 1985: 77, en Kinnunen et al. 2010). Este conflicto inter-roles funciona bidireccionalmente, es decir, el trabajo 
puede interferir con la familia (conflicto trabajofamilia, en inglés conocido con las siglas WFC) y la familia puede interferir con el trabajo (conflicto familia-trabajo, en inglés conocido con las siglas FWC).

La literatura evidencia que el conflicto trabajofamilia está asociado con varios resultados individuales negativos, así como con efectos en la relación empleado-organización (Carlson y Perrew 1999, en Darcy y McCarthy 2007; Allen et al. 2000; Kossek y Ozeki 1998).

En efecto, el conflicto entre la vida laboral y familiar puede tener varios resultados. En una revisión exhaustiva del tema, Allen et al. (2000) presenta las consecuencias relacionadas con el conflicto trabajo-familia, agrupándolas en tres categorías. La primera de ellas, compuesta por aquellos conflictos relacionados con efectos en el trabajo (tales como la satisfacción laboral, el compromiso con la organización, el desempeño en el trabajo). En una segunda categoría, se ubican las consecuencias no relacionadas con el trabajo (tales como la satisfacción con la vida, la satisfacción marital, la satisfacción del placer) $\mathrm{y}$, en una tercera categoría, las consecuencias relacionadas con el estrés (tales como la depresión, el agotamiento, la tensión psicológica general).

En cuanto a la tensión experimentada por los trabajadores como resultado del conflicto percibido, y su efecto en el trabajo, para Thompson et al. (1999, en O'Brien y Hayden 2008) si las empresas no impulsan las prácticas para balancear la relación trabajo-familia estarán contribuyendo con mayores tensiones en las vidas personales de su equipo humano, lo que luego afectará su concentración y rendimiento en el trabajo. Más allá de la satisfacción laboral, un aumento del conflicto trabajo-familia estará relacionado también con un aumento en las intenciones de cambio de empleo (Greenhaus et al. 2001; Kelloway et al. 1999; en Mitchelson 2009).

Entre las consecuencias relacionadas con los cambios en la actitud personal, como producto del conflicto trabajo-familia, los estudios reportan un menor nivel de satisfacción de la vida y del trabajo (Kossek y Ozecki 1998), una
Sandra Vera Linares - Amaia Arizkuren Eleta - Tanuja Agarwala

menor satisfacción marital (Judge et al. 2006; Matthews at al. 1996), y una menor satisfacción de la familia (Bedeian et al. 1988).

Existe abundante bibliografía que relaciona los efectos de este tipo de conflictos con el estrés (Kelloway et al. 1999; Parasurman y Simmers 2001), se relaciona también con el aumento de la depresión y la ansiedad (Frone 2000; Grzywacz y Bass 2003), con los trastornos de ansiedad, trastornos del humor, trastornos de abuso de sustancias (Frone 2000), con las quejas sobre la salud física e hipertensión (Frone at al. 1997), e, incluso, con el consumo de alimentos grasos (Allen y Armstrong 2006).

En este mismo sentido, se han encontrado evidencias de una correlación entre conflicto trabajo-familia en las tres dimensiones descritas. Este es el caso del efecto del conflicto en el estrés laboral; estrés familiar; intenciones de rotación en el empleo; abuso de sustancias no naturales; menor satisfacción familiar; marital, laboral, y de vida y, finalmente, afectación del compromiso organizacional y del rendimiento (Allen et al. 2000; Eby et al. 2005; Kossek y Ozeki 1998 y Netemeyer et al. 1996).

No obstante lo anterior, cuando un trabajador enfrenta un fuerte conflicto trabajo-familia y cuenta con apoyo social de directivos y colegas, reducirá la tensión resultante del conflicto. En este sentido, los trabajadores que cuentan con jefes directos apoyadores han reportado menor conflicto trabajo-familia (Goff et al. 1990). Por el contrario, se han encontrado altos niveles de conflicto trabajo-familia cuando hay falta de apoyo social (Greenhaus y Beutell 1985).

Siguiendo esta línea de pensamiento se ha planteado la siguiente hipótesis:

Hipótesis 1: Una cultura organizacional que apoya el balance trabajo-familia está relacionada negativamente con el nivel del conflicto que experimentan los trabajadores.

Cultura organizacional que apoya el balance trabajo-familia y la voluntad de permanencia en la empresa

Senge (1998) resalta la necesidad de que en las organizaciones se tome importancia al equilibrio de la vida laboral y personal para potenciar el 
compromiso de los trabajadores y, de ese modo, incrementar las capacidades de la organización. Para Goulding y Kerslake (1996), el origen de las prácticas de trabajo flexible se debe, entre otras razones, a la retención de trabajadores valiosos que tienen expectativas en la empresa, en relación con la atención de este tema. Para Wise y Bond (2003, en O’Brien y Hayden 2008), estas prácticas contribuyen a que la empresa sea considerada una empleadora atractiva y preocupada por temas de inclusión $\mathrm{y}$, asimismo, incrementan la retención del talento, debido a que se logra resguardar la voluntad de permanencia en la empresa.

En este mismo sentido, Grover y Crooker (1995) encontraron que los trabajadores en empresas con beneficios de equilibrio trabajo-familia mostraban mayores niveles de compromiso con la organización y expresaban menos intenciones de rotación, independientemente de si el trabajador individual se beneficiaba de la política. Según estos autores, los beneficios de balance trabajofamilia presentan una influencia positiva sobre el apego de los trabajadores a la organización, ya que significan preocupación corporativa para los trabajadores y sus familias.

Para Kisilevitz y Bedington (2009) los programas de balance trabajo-familia implementados efectivamente ofrecen un sustancial retorno de la inversión a través de un mayor compromiso y una reforzada proposición de valor del empleo. Chinchilla et al. (2010) afirman que las empresas que desarrollen una cultura de responsabilidad frente a la preocupación del trabajador por su familia podrán agregar atributos a su ventaja competitiva al atraer, retener y promover a las personas más talentosas. No obstante lo anterior, refieren que no bastará con que las empresas desarrollen un listado de prácticas que promuevan el balance de la vida laboral y personal, sino que tendrán que preocuparse por establecer las condiciones básicas para que la cultura corporativa asuma los valores que promueven este balance, si lo que se quiere es que las prácticas diseñadas sean adoptadas por los individuos. Es decir, se trata de crear una cultura que realmente apoye el uso de estas prácticas, lo que requiere de transformaciones
Sandra Vera Linares - Amaia Arizkuren Eleta - Tanuja Agarwala

más profundas en los valores organizacionales y de los miembros de la organización. Estos autores sostienen que la teoría del intercambio social establece que la reacción natural de un trabajador ante el beneficio que recibe de su empresa es sentir una obligación recíproca. Es de esperar que los trabajadores que conocen las prácticas de balance trabajo-familia que ofrecen sus empresas generen mayor compromiso hacia la empresa y se esfuercen más. Pueden incluso llegar a sentir que en otra organización no contarían con los mismos beneficios y, en consecuencia, analizarán el costo de dejar la organización.

\section{Conflicto en el balance trabajo-familia y la voluntad de permanencia en la empresa}

El balance trabajo-familia refleja la integración de las demandas del trabajo con los roles en la familia, mientras que el conflicto trabajo-familia representa incompatibilidades entre el trabajo y las responsabilidades familiares por falta de recursos, tales como el tiempo y la energía (Kahn et al., 1964 en Gropel y Kuhl 2009).

Diversos autores han identificado varios efectos del conflicto trabajo-familia, discutidos en secciones previas. En particular, las principales referencias al efecto final o más radical en la situación de la relación laboral se expresan en un cambio en la voluntad de permanencia en la empresa por parte de los trabajadores lo que, a su vez, se traduce en la rotación en el empleo o retiro del trabajo (Allen et al. 2000). En efecto, según sostienen Heraty et al. (2008), en el plano laboral, la existencia de conflictos trabajo-familia llevará finalmente al aumento del ausentismo y de la rotación laboral. Para Streich et al. (2007), esto puede explicarse por la reducción del nivel de compromiso afectivo con la organización.

En función a la literatura revisada, se plantean las hipótesis $2 a, 2 b$ y 3 , mostradas a continuación:

\footnotetext{
Hipótesis 2a: El conflicto trabajo-familia (WFC) está relacionado negativamente con la voluntad de permanencia en la empresa por parte del trabajador.

Hipótesis 2b: El conflicto familia-trabajo (FWC) está relacionado negativamente con la voluntad de permanencia en la empresa por parte del trabajador.

Hipótesis 3: Los conflictos trabajo-familia (WFC) y familia-trabajo (FWC) y la cultura que apoya el equilibrio trabajo-familia se relacionan con la voluntad de permanencia en la empresa, negativa y positivamente, respectivamente.
} 


\section{IV) Metodología}

Esta investigación estuvo dirigida a tres sectores relevantes en el país, por el nivel de empleo formal que generan. Estos sectores fueron: el financiero, el industrial y el de telecomunicaciones. Según el Ministerio de Trabajo y Promoción del Empleo (2010), a 2009, del total de la Población Económicamente Activa Ocupada, la industria concentra el 10.2\% del empleo, mientras que el sector servicios el $32 \%)$. Las encuestas se dirigieron a empresas consideradas grandes (más de 500 trabajadores). Se aplicaron un total de 96 cuestionarios a trabajadores de empresas peruanas (31 encuestas del sector financiero, 29 del sector industrial y 36 del sector de telecomunicaciones). La encuesta fue enviada de manera virtual y fue respondida voluntariamente por los trabajadores. El 53\% de los encuestados fueron mujeres y el $47 \%$ fueron hombres. La edad promedio de los encuestados fue 37 años. El cuestionario utilizado constó de 20 ítems y abordó los tres constructos bajo estudio: cultura, conflicto y voluntad de permanencia. En el Apéndice 1 se listan los ítems empleados en el estudio.

\section{Cultura}

El constructo Cultura que Apoya el Balance Trabajo-Familia fue consultado a través de 6 ítems que responden a los tópicos más citados en la literatura previa. Se tomó como base el cuestionario desarrollado por Thompson et al, (1999). Este constructo fue validado a través de un análisis factorial ${ }^{1}$, cuya solución obtuvo un Alpha de Cronbach de 0.894. Para la calificación de estos ítems se utilizó una escala Likert de 5 puntos.
Sandra Vera Linares - Amaia Arizkuren Eleta - Tanuja Agarwala

\section{Conflicto}

En concordancia con la literatura existente, a través del análisis factorial se identificaron dos componentes del Conflicto en el Balance Trabajo-Familia. Estos dos componentes fueron el Conflicto Trabajo-Familia (WFC) y el Conflicto Familia-Trabajo (FWC). El conflicto trabajofamilia se midió utilizando 4 ítems y el conflicto familia-trabajo se midió utilizando 6 ítems, estos se basaron en la escala desarrollada por Carlson et al. (2000)2. El Alpha de Cronbach para el factor Conflicto Trabajo-Familia fue de 0.874 y para el factor Conflicto Familia-Trabajo fue de 0.921, lo cual confirmó la consistencia interna en cada caso. Para todas las mediciones se utilizó una escala Likert de 5 puntos.

\section{Permanencia en la empresa}

El análisis factorial determinó que son cuatro los ítems que miden la Voluntad de Permanencia en la Empresa ${ }^{3}$. Una versión corta del Cuestionario de Compromiso Organizacional (Allen y Meyer 1990) fue utilizada. El Alpha de Cronbach obtenido fue de 0.843 . Se utilizó una escala de Likert de 5 puntos.

\section{V) Análisis de datos}

Los datos fueron analizados utilizando el paquete estadístico SPSS versión 20. Se pueden apreciar los reportes obtenidos en el Apéndice 2.

\section{VI) Resultados}

Según lo esperado, los resultados del análisis de regresión confirmaron la hipótesis $1 \mathrm{e}$ indicaron que la cultura que apoya el balance

1. Las comunalidades fueron todas mayores a 0.5 lo que indica que todos los ítems aportaron a la estructura factorial. La varianza total explicada fue de 65.47\%. La matriz de componentes muestró que cada ítem cargaba de manera alta en el factor identificado.

2. En ambos casos, las comunalidades fueron todas mayores a 0.5, lo que indica que todos los ítems aportaron a la estructura factorial. La varianza total explicada fue de $72,79 \%$ y $72.27 \%$, respectivamente. En ambos casos, la matriz de componentes mostró que cada ítem cargaba de manera alta en el factor identificado.

3. Las comunalidades de los ítems indicaron la contribución de cada uno a la estructura factorial. La varianza total explicada fue de 68,36\%. La matriz de componentes muestró que las cargas de cada ítem eran altas en el mismo factor, indicando la medición de un mismo concepto. 
trabajo-familia predice el nivel de conflicto que experimenta el trabajador en este tema. En este sentido, el conflicto experimentado por el trabajador guarda una relación negativa con una cultura que apoya el balance trabajo-familia.

$$
\begin{aligned}
& \text { Conflicto }=37,975-0.658 \text { cultura } \\
& (R 2=0.184, p<0,05)
\end{aligned}
$$

Las hipótesis $2 a$ y $2 b$ fueron analizadas y confirmadas a través de una regresión múltiple, donde las variables referidas al conflicto trabajo-familia (WFC) y conflicto familia-trabajo (FWC) lograron una contribución significativa para estimar la voluntad de permanencia del trabajador en la empresa.

Voluntad de permanencia $=2810-0,282$ WFC $-0,228$ FWC ( $R 2$ corregido $=0.226, P=0.000$ )

Finalmente, la hipótesis 3 fue confirmada parcialmente mediante una regresión múltiple que permitió evidenciar que dos variables predicen la voluntad de permanencia del trabajador en el empleo: la cultura de apoyo al balance trabajo-familia y el conflicto familiatrabajo (FWC). Según ello, la relación entre el conflicto familia-trabajo (FWC) y la voluntad de permanencia en la empresa resultó negativa y la relación entre cultura de apoyo al balance trabajo-familia y la voluntad de permanencia en la empresa resultó positiva. La variable conflicto trabajo-familia (WFC) no resultó significativa.

Voluntad de permanencia $=-6,274-0,232$ FWC

$+0,310$ cultura ( $R 2$ corregido $0,324, p=0.000$ )

\section{VII) Conclusiones}

Los hallazgos de esta investigación evidencian, en línea con la literatura previa (Kahn et al., 1964 en Gropel y Kuhl 2009), que el conflicto trabajofamilia (WFC) y el conflicto familia-trabajo (FWC) constituyen dos factores que configuran el concepto general de conflicto en el balance entre la vida laboral y la vida familiar. Más aún, este estudio muestra que, como afirman Allen et al. (2000) y Heraty et al. (2008), los conflictos que el trabajador experimenta por las presiones de sus roles en el trabajo y en la vida familiar afectan su voluntad de permanencia en la empresa.
Sandra Vera Linares - Amaia Arizkuren Eleta - Tanuja Agarwala

Como sugieren los diversos autores citados en este artículo, más allá de contar con políticas que promuevan el balance trabajo-familia, la existencia de una cultura corporativa que apoye el uso de estas prácticas resulta relevante para que el trabajador no se sienta hasta el punto de cuestionar su permanencia en la empresa.

Finalmente, según se muestra en esta investigación, cuando se busca predecir la voluntad de permanencia del trabajador en su empleo y, además de considerar los dos tipos de conflicto mencionados, se incorpora la cultura como un elemento que apoya el balance trabajofamilia; el conflicto trabajo-familia (WFC) deja de resultar significativo, aunque persiste la relación negativa entre el conflicto familia-trabajo (FWC) y la voluntad de permanencia en la empresa por parte del trabajador. La investigación realizada contribuye a la gestión eficiente del equilibrio trabajo-vida en las empresas peruanas puesto que evidencia que no basta con ofrecer beneficios que propicien el balance entre el ámbito familiar y laboral si es que se desea retener a los colaboradores. Lo que se debe promover es una cultura que fomente la utilización de este tipo de prácticas puesto que este apoyo tendrá un efecto significativo en la voluntad de permanencia en la empresa. El estudio revela que el conflicto de la familia en el trabajo (FWC) es el que podría llevar a los trabajadores peruanos a tomar la decisión de renunciar, por lo que una cultura de apoyo se hace necesaria también en este sentido.

Estos hallazgos sugieren la ejecución de pruebas confirmatorias que permitan explicar si una cultura que apoya el balance trabajo-familia reduce el impacto de los conflictos que surgen en la relación familiar del trabajador, como producto de la influencia de su rol laboral. 


\section{BIBLIOGRAFÍA}

ALLEN, T. D., y J. Armstrong

2006 «Further examination of the link between work-family conflict and physical health: The role of health-related behaviors». American Behavioral Scientist, 49, 1204-21.

ALLEN, T. D., D. E. Herst, C. S. Bruck y M. Sutton 2000 "Consequences associated with work-to-family conflict: A review and agenda for future research». Journal of Occupational Health Psychology, 5, 278-308.

ALLEN, N. J. y J. P. Meyer

1990 «The measurement and antecedents of affective, continuance and normative commitment to the organization». Journal of Oc- cupational Psychology 63.

ALLES, $M$.

2000 Mujeres, trabajo y autoempleo. Otro camino frente al desempleo y desarrollo de carrera. Barcelona: Granica.

BEDEIAN, A.G., B. G. Burke y R. G. Moffett

1988 «Outcomes of work-family conflict among married male and female professionals». Journal of Management, 14, 475-91.

BURKE, R.

2010 «Do managerial men benefit from organizationalvaluessupportingwork-personal life balance?». Gender in Management: An International Journal, 25(2), 91-9.

CARLSON, D. S., K. M. Kacmar, y L. J. Williams

2000 "Construction and initial validation of a multidimensional measure of work-family conflict». Journal of Vocational Behavior, 56, 249-76.

CHINCHILLA, N., M. Las Heras y E. Torres

2010 «Work-Family Balance: A Global Challenge». Chinchilla, N., Las Heras, M. y Masuda, A.D. (editoras). Balancing Work and Family. Massachusetts: HRD Press, 7-22.

DARCY, C., y A. McCarthy

2007 «Work-family conflict An exploration of the differential effects of a dependent child's age on working parents». Journal of European Industrial Training, 31(7), 530-49.

EBY, L. T., W. J. Casper, A. Lockwood, C. Bordeaux y A. Brinley

2005 «Work and Family Research in IO/ OB: Content Analysis and Review of the Literature (19802002) ». Journal of Vocational Behavior, 66, 124-97.
Sandra Vera Linares - Amaia Arizkuren Eleta - Tanuja Agarwala

FRONE, M. R., M. Russell y M. L. Cooper

1997 «Relation of work-family conflict to health outcomes: A four-year longitudinal study of employed parents». Journal of Occupational and Organizational Psychology, 70, 325-35.

FRONE, M. R.

2000 «Work-family conflict and employee psychiatric disorders: The national comorbidity study». Journal of Applied Psychology, 85, 888-95.

GREENHAUS, J. H., y N. J. Beutell

1985 «Sources of conflict between work and family roles». Academy of Management Review, 10(1), 76-85.

GREENHAUS, J. H., S. Parasuraman y K. M. Collins

2001 «Career involvement and family involvement as moderators of relationships between work-family conflict and withdrawal from a profession». Journal of Occupational Health Psychology, 6, 91-100.

GOFF, S., M. Mount, M, y R. Jamison

1990 «Employer Supported Child Care, Work/ Family Conflict, and Absenteeism: A Field Study». Personnel Psychology, 43, 693-709.

GOULDING, A. y E. Kerslake

1996 «Flexible working in libraries: profit and potential pitfalls». Library Management, 17(2), 8-16.

GROPEL, P., y J. Kuhl

2009 «Work-life balance and subjective well-being: The mediating role of need fulfilment». British Journal of Psychology, 100, 365-75.

GROVER, S. L., y K. J. Crooker

1995 «Who appreciates family-responsive human resource policies: The impact of family-friendly policies on the organizational attachment of parents and non-parents». Personnel Psychology, 48, 271-88.

GRZYWACZ, J. G. y B. L. Bass

2003 «Work, family and mental health: Testing different models of work-family fit». Journal of Marriage and Family, 65, 248-62.

HERATY, N., M. Morley y J. Cleveland

2008 «Complexities and challenges in the workfamily interface». Journal of Managerial psychology, 23(3), 209-14.

JUDGE, T. A., R, Ilies y B. A. Scott

2006 «Work-family conflict and emotions: Effects at work and at home». Personnel Psychology, 59, 779-814. 
KABANOFF, B., R. Waldersee y M. Cohen

1995 «Espoused values and organizational change themes». Academy of Management Journal, 38(4), 1075-104.

KELLOWAY, E. K., B. H. Gottlieb y L. Barham

1999 «The source, nature, and direction of work and family conflict: A longitudinal investigation». Journal of Occupational Health Psychology, 4, 337-46.

KINNUNEN, U., T. Feldt, S. Mauno y J. Rantanen

2010 «Interface between work and family: A longitudinal individual and crossover perspective». Journal of Occupational and Organizational Psychology, 83(1), 119-37.

KISILEVITZ, M., y T. Bedington

2009 «Managing Work-Life Balance Programs». Benefits \& Compensation Digest, 30-4.

KOSSEK, E., y C. Ozeki

1998 «Work- Family Conflict, Policies, and the Job - Life Satisfaction Relationship: A Review and Directions for Organizational BehaviourHuman Resources Research». Journal of Applied Psychology, 83, 139-49.

LEWIS, S.

1999 "How to voice the needs to reconcile work and family in the workplace? European diversities. Combining work and family in different settings of working life, family life and culture». Seminar Report, 43-51. Helsinki: Stakes.

LINGARD, H., K. Brown, L. Bradley, C. Bailey y K. Townsend

2007 «Improving Employees’ Work-Life Balance in the Construction Industry: Project Alliance Case Study». Journal of Construction Engineeringand Management, 807-15.

MATTHEWS, L.S., R. D. Conger y K. A. S. Wickrama

1996 «Work-family conflict and marital quality: Mediating processes». Social Psychology Quarterly, 59, 62-79.

MAUNO, S., U. Kinnunen y S. Piitulainen, S.

2005 «Work-family culture in four organizations in Finland». Community, Work and Family, 8(2), 115-40.

MINISTERIO DE TRABAJO Y PROMOCIÓN DEL EMPLEO

2010 Informe Anual. El Empleo en el Perú y Lima Metropolitana 2009. En: www.mintra.gob.pe.
Sandra Vera Linares - Amaia Arizkuren Eleta - Tanuja Agarwala

MITCHELSON, J.

2009 «Seeking ttbe perfect balance: Perfectionism and work-family conflict». Journal of Occupational and Organizational Psychology, 82, 349-67.

NETEMEYER, R.G., J. Boles y R. McMurrian

1996 «Development and Validation of Work-family Conflict and Family-work Conflict Scales». Joumal of Applied Psychology, 81, 400-9.

O'BRIEN, T. y H. Hayden

2008 «Flexible work practices and the LIS sector: balancing the needs of work and life?». Library Management, 29(3), 199-228.

PARASURAMAN, S. y Simmers, C.

2001 «Type of employment, work-family conflict and wellbeing: A comparative study». Journal of Organizational Behavior, 22, 551-68.

SENGE, $P$.

1998 La quinta disciplina. Buenos Aires: Granica.

STREICH, M., W. Casper y A. Salvaggio

2008 «Examining couple agreement about workfamily conflict». Journal of Managerial Psychology, 23(3), 252-72.

THOMPSON, C.A., L. L. Beauvais y K. S. Lyness

1999 «When work-family benefits are not enough: The influence of work-family culture on Benefit utilization, organizational attachment, and work-family conflict». Journal of Vocational Behavior, 54, 392-415. 


\section{Apéndices}

\section{Apéndice 1}

\section{ÍTEMS DEL CUESTIONARIO}

\section{CULTURA}

- En general, la dirección de mi organización es bastante complaciente respecto a las necesidades personales y familiares de los empleados.

- En mi organización, los empleados son alentados para lograr un equilibrio entre su trabajo y vida personal y familiar.

- En general, la dirección de mi organización muestra sensibilidad y comprensión hacia las responsabilidades de los empleados en relación al cuidado de los ancianos.

- En general, la dirección de mi organización muestra sensibilidad y comprensión hacia las responsabilidades de los empleados en relación al cuidado de los niños.

- En el caso de un conflicto entre las exigencias laborales y familiares, la dirección de mi organización es muy comprensiva cuando los empleados tienen que poner su vida personal y familiar en primer lugar.]

- En mi organización, los empleados pueden equilibrar su trabajo y vida personal y familiar.

\section{CONFLICTO TRABAJO-VIDA PERSONAL}

- Después del trabajo, vuelvo a casa demasiado cansado para hacer algunas de las cosas que me gustaría hacer, como por ejemplo, pasar tiempo con mi familia, o disfrutar de mis aficiones e intereses.]

- Debido a la presión del trabajo, estoy a menudo demasiado estresado para hacer las cosas que me gusta cuando llego a casa.

- Estoy a menudo demasiado preocupado por el trabajo, incluso cuando estoy en casa.

- El estrés y la tensión de mi trabajo a menudo me hacen estar irritable cuando llego a casa después de trabajar.

\section{CONFLICTO VIDA PERSONAL-TRABAJO}

- La cantidad de tiempo que dedico a mi vida personal y familiar y las responsabilidades del hogar, a menudo interfieren con las responsabilidades de mi trabajo.

- Estoy a menudo demasiado cansado en mi trabajo a causa de mi vida familiar y personal.

- Siento que no tengo suficiente tiempo para desarrollar todo mi potencial en el trabajo a causa de la demanda de mi vida personal, como tener que pasar tiempo con amigos, familia, etc.

- Debido al estrés en mi vida personal / familiar, a menudo me preocupo de los asuntos personales y familiares, incluso cuando estoy en la oficina o en el trabajo.

- El estrés y la tensión de mi vida personal o familiar (no laboral), interfiere con mi vida laboral.

- Debido a todas las presiones de mi vida personal y las responsabilidades familiares, a veces me resulta difícil hacer bien mi trabajo.

\section{VOLUNTAD DE PERTENENCIA}

- No me siento como «parte de la familia» en mi empresa actual.

- No me siento emocionalmente unido a mi empresa actual.

- En este momento, quedarme en mi compañía actual es una cuestión de necesidad más que de deseo.

- No quiero seguir en mi compañía actual. 
Tabla 1. Regresión para Hipótesis 1

Variables introducidas/eliminadas ${ }^{\text {a }}$
\begin{tabular}{|c|c|c|r|}
\hline Modelo & Variables introducidas & Variables eliminadas & Método \\
\hline 1 & Cultura $^{b}$ &. & Introducir \\
\hline
\end{tabular}

a. Variable dependiente: CONFLICTO_TOTAL.

b. Todas las variables solicitadas introducidas.

\section{ANOVAa $^{a}$}

\begin{tabular}{|c|c|c|c|c|c|}
\hline Modelo & Suma de cuadrados & $\mathrm{gl}$ & Media cuadrática & $\mathrm{F}$ & Sig. \\
\hline 1 Regresión & 940,925 & 1 & 940,925 & 21,231 &, $000^{\mathrm{b}}$ \\
\hline Residual & 4166,033 & 94 & 44,320 & & \\
\hline Total & 5106,958 & 95 & & & \\
\hline
\end{tabular}

a. Variable dependiente: CONFLICTO TOTAL.

b. Variables predictoras: (Constante), Cultura.

Tabla 2. Regresión para Hipótesis 2a y 2b

\section{Variables introducidas/eliminadas ${ }^{a}$}

\begin{tabular}{|c|c|c|c|}
\hline Modelo & Variables introducidas & Variables eliminadas & Método \\
\hline 1 & $\begin{array}{c}\text { Conflicto_VW, } \\
\text { Conflicto_WV }\end{array}$ & & Introducir \\
\hline
\end{tabular}

a. Variable dependiente: REC_VoIPERMANENCIA.

b. Todas las variables solicitadas introducidas.

\begin{tabular}{|c|c|c|c|c|}
\hline \multicolumn{5}{|c|}{ Resumen del modelo } \\
\hline Modelo & $\mathrm{R}$ & R cuadrado & $\begin{array}{c}\text { R cuadrado } \\
\text { corregida }\end{array}$ & $\begin{array}{l}\text { Error típ. de la } \\
\text { estimación }\end{array}$ \\
\hline 1 &, $429^{a}$ & , 184 & , 176 & 6,65729 \\
\hline
\end{tabular}

a. Variables predictoras: (Constante), Cultura.

\section{Coeficientes $^{a}$}

\begin{tabular}{|c|c|c|c|c|c|c|}
\hline \multirow{2}{*}{\multicolumn{2}{|c|}{ Modelo }} & \multicolumn{2}{|c|}{$\begin{array}{l}\text { Coeficientes no } \\
\text { estandarizados }\end{array}$} & \multirow{2}{*}{$\begin{array}{c}\begin{array}{c}\text { Coeficientes } \\
\text { tipificados }\end{array} \\
\text { Beta }\end{array}$} & \multirow[t]{2}{*}{$\mathrm{t}$} & \multirow[t]{2}{*}{ Sig. } \\
\hline & & B & Error típ. & & & \\
\hline \multirow[t]{2}{*}{1} & (Constante) & 37,975 & 2,945 & 12,894 & &, 000 \\
\hline & Cultura &,- 658 & 143 &,- 429 & $-4,608$ &, 000 \\
\hline
\end{tabular}

\section{Resumen del modelo}

\begin{tabular}{|c|c|c|c|c|}
\hline Modelo & $\mathrm{R}$ & $\mathrm{R}$ cuadrado & $\begin{array}{c}\mathrm{R} \text { cuadrado } \\
\text { corregida }\end{array}$ & $\begin{array}{c}\text { Error típ. de la } \\
\text { estimación }\end{array}$ \\
\hline 1 &, $492^{\mathrm{a}}$ &, 242 &, 226 & 3,26973 \\
\hline
\end{tabular}

a. Variables predictoras: (Constante), Conflicto_VW, Conflicto_WV.

\section{ANOVA $^{\mathrm{a}}$}

\begin{tabular}{|c|c|c|c|c|c|}
\hline Modelo & Suma de cuadrados & $\mathrm{gl}$ & Media cuadrática & $\mathrm{F}$ & Sig. \\
\hline 1 Regresión & 317,717 & 2 & 158,858 & 14,859 &, $000^{\mathrm{b}}$ \\
\hline $\begin{array}{r}\text { Residual } \\
\text { Total }\end{array}$ & $\begin{array}{c}994,273 \\
1311,990\end{array}$ & $\begin{array}{l}93 \\
95\end{array}$ & 10,691 & & \\
\hline
\end{tabular}

a. Variable dependiente: REC_VoIPERMANENCIA

b. Variables predictoras: (Constante), Conflicto_VW, Conflicto_WV.

\begin{tabular}{|c|c|c|c|c|c|}
\hline \multicolumn{6}{|c|}{ Coeficientes $^{a}$} \\
\hline Modelo & \multicolumn{2}{|c|}{$\begin{array}{l}\text { Coeficientes no } \\
\text { estandarizados }\end{array}$} & $\begin{array}{l}\text { Coeficientes } \\
\text { tipificados }\end{array}$ & $\mathrm{t}$ & Sig. \\
\hline & B & Error típ. & Beta & & \\
\hline 1 (Constante) & 2,810 & 1,245 & & 2,258 &, 026 \\
\hline Conflicto_WV &,- 282 & ,094 &,- 286 & $-3,013$ & ,003 \\
\hline Conflicto_WV &,- 282 & ,094 &,- 286 & $-3,013$ &, 003 \\
\hline
\end{tabular}

a. Variable dependiente: REC_VoIPERMANENCIA. 
Tabla 3. Regresión para Hipótesis 3

Variables introducidas/eliminadas ${ }^{\text {a }}$
\begin{tabular}{|c|c|c|c|}
\hline Modelo & Variables introducidas & Variables eliminadas & Método \\
\hline 1 & $\begin{array}{c}\text { Cultura, } \\
\text { Conflicto_VW, } \\
\text { Conflicto_WV }\end{array}$ & & \\
& & & Introducir \\
\hline
\end{tabular}

a. Variable dependiente: REC VoIPERMANENCIA.

b. Todas las variables solicitadas introducidas.

\section{ANOVA $^{\mathrm{a}}$}

\begin{tabular}{|c|c|c|c|c|c|}
\hline Modelo & Suma de cuadrados & $\mathrm{gl}$ & Media cuadrática & $\mathrm{F}$ & Sig. \\
\hline 1 Regresión & 452,959 & 3 & 150,986 & 16,170 &, $000^{\mathrm{b}}$ \\
\hline Residual & 859,031 & 92 & 9,337 & & \\
\hline Total & 1311,990 & 95 & & & \\
\hline
\end{tabular}

a. Variable dependiente: REC_VoIPERTENEC.

b. Variables predictoras: (Constante), Cultura, Conflicto_VW, Conflicto_WV.

\section{Resumen del modelo}

\begin{tabular}{|c|c|c|c|c|}
\hline Modelo & $\mathrm{R}$ & $\mathrm{R}$ cuadrado & $\begin{array}{c}\text { R cuadrado } \\
\text { corregida }\end{array}$ & $\begin{array}{c}\text { Error típ. de la } \\
\text { estimación }\end{array}$ \\
\hline 1 &, $588^{\mathrm{a}}$ &, 345 &, 324 & 3,05570 \\
\hline
\end{tabular}

a. Variables predictoras: (Constante), Cultura, Conflicto_VW, Conflicto_WV.

\begin{tabular}{|c|c|c|c|c|c|}
\hline \multicolumn{6}{|c|}{ Coeficientes $^{a}$} \\
\hline Modelo & \multicolumn{2}{|c|}{$\begin{array}{l}\text { Coeficientes no } \\
\text { estandarizados }\end{array}$} & $\begin{array}{c}\text { Coeficientes } \\
\text { tipificados }\end{array}$ & $\mathrm{t}$ & Sig. \\
\hline & B & Error típ. & Beta & & \\
\hline 1 (Constante) & $-6,274$ & 2,655 & & $-2,363$ &, 020 \\
\hline Conflicto_WV &,- 047 & , 107 &,- 048 &,- 439 & ,662 \\
\hline Conflicto_WV &,- 232 &, 063 &,- 326 & $-3,674$ &, 000 \\
\hline Cultura & ,310 &, 081 & ,399 & 3,806 &, 000 \\
\hline
\end{tabular}

a. Variable dependiente: REC VoIPERMANENCIA. 